\title{
Exercise for Cardiovascular Disease Patients: The New Normal Life in COVID-19 Pandemic
}

\author{
Ruhollah Nourian (iD) ${ }^{1}$, Sepideh Niyazi (iD) ${ }^{2}$, Mahboubeh Ghayour Najafabadi ${ }^{3}$, Amir Sobhrakhshan \\ Khah (iD ${ }^{4,5,{ }^{*}}$ and Mohammad Hossein Pourgharib Shahi ${ }^{6,2}$ \\ ${ }^{1}$ Sports Medicine Research Center, Neuroscience Institute, Tehran University of Medical Sciences, Tehran, Iran \\ ${ }^{2}$ Department of Sports and Exercise Medicine, Shariati Hospital, Tehran University of Medical Sciences, Tehran, Iran \\ ${ }^{3}$ Department of Motor Behavior, Faculty of Physical Education and Sport Sciences, University of Tehran, Tehran, Iran \\ ${ }^{4}$ Sepehr Heart Center, Baharloo Hospital, Tehran University of Medical Sciences, Tehran, Iran \\ ${ }^{5}$ Department of Cardiology, School of Medicine, Tehran University of Medical Sciences, Tehran, Iran \\ ${ }^{6}$ Department of Sports and Exercise Medicine, School of Medicine, Tehran University of Medical Sciences, Tehran, Iran \\ "Corresponding author: Sepehr Heart Center, Baharloo Hospital, Tehran University of Medical Sciences, Tehran, Iran. Email: rakhshankhah.md@gmail.com
}

Received 2020 December 09; Revised 2021 March 08; Accepted 2021 April 16.

Keywords: COVID-19, Cardiovascular Disease, Cardiac Rehabilitation

\section{Dear editor,}

In December 2019, the first report of the new Coronavirus disease 2019 (COVID-19) was received, with over seven hundred thousand deaths worldwide to date. Many of the patients with chronic illnesses, cardiovascular disease (CVD), are at risk of higher mortality and morbidity (1). In addition, the World Health Organization (WHO) has suggested that people should avoid unnecessary outdoor activities and keep a social distance of at least 6 feet from other people and stay at home, especially for people with underlying chronic diseases (2). A significant problem with this approach, however, is the harmful effects of physical inactivity that may cause psychological and physical issues, especially in CVD patients. It has been established that beneficial metabolic and CV adaptations in response to exercise can be lost only after two weeks of inactivity and can lead to a decline in functional capacity and have detrimental effects on risk factors for coronary artery disease. There is an uncertain future about how long COVID-19 will be there. Therefore adaptation to this situation is an important consideration (3). This is a brief look at what could be a new way to approach having a healthy lifestyle, as well as safe alternatives to cardiac rehabilitation centers at these trying times.

\section{The new normal life for CVD patients}

\section{A more active life:}

A wide range of illustrated exercises such as videos or mobile applications for aerobic, strengthening, core stability, and balance exercises are available throughout the Internet. However, the safety of these exercises should be considered by health care providers before their prescription to CVD patients (Table 1). The outcomes of exercise are not limited to better functional capacity. It also helps the patients in maintaining a positive mood, better stress control, reducing CVD risk factors, and a higher quality of life (3). In order to enable patients to keep active, we recommend low-intensity exercises that are defined as a metabolic equivalent (MET) of less than 3, as well as a rating of perceived exertion (RPE) of between $8-10$ of 20 or 1 - 2 of 10 scales. Slow walking, bird watching, watering plants, Hatha yoga and stretching exercises, darts, billiard, and fishing are proper examples for this aim (4). Considering CV diseases and other comorbidities, it seems that stable CVD patients can freely perform the suggested activities without any restriction.

\section{Moving towards a more physically active life from home:}

What is the solution for CVD patients looking for a more active lifestyle while staying at home? It could be the home-based cardiac rehabilitation strategy previously stated as a practical solution for mild to moderate risk CVD patients by the American Association of Cardiovascular and Pulmonary Rehabilitation (AACVPR), American Heart Association (AHA), and American College of Cardiology (ACC) (5). We suggest a virtual consult for risk stratification $(5,9)$ to find out the level of risk, comorbidities, drug history, and physical activity profile. While the patients rated as low to moderate risk for cardiac rehabilitation are recommended to begin home-based protocols, highrisk patients need to exercise more cautiously; performing 


\begin{tabular}{ll}
\hline Table 1. Safety Considerations for Home-Based Exercise in CVD Patients \\
\hline Safety & Values \\
\hline E-learning & $\begin{array}{l}\text { Registration of consultant; Proficiency of } \\
\text { consultant; Face to face education/consultation; } \\
\text { Illustrated exercises (photo/video) (5, 6) }\end{array}$ \\
\hline Assessment of patients & $\begin{array}{l}\text { Risk stratification: Online form (7, 8); } \\
\text { Phone/video call (5) }\end{array}$ \\
\hline Environmental & $\begin{array}{l}\text { Proper place for exercise; Clean facilities; } \\
\text { Avoiding extreme weather (hot and humid) (5) }\end{array}$ \\
\hline Intensity of exercises & $\begin{array}{l}\text { Using RPE instead of heart rate-based intensity } \\
\text { prescription; Teaching warning signs to stop the } \\
\text { exercise (exertional chest pain, excessive fatigue, } \\
\text { shortness of breath, lightheadedness) (6) }\end{array}$ \\
\hline Medical issues & $\begin{array}{l}\text { Drug history; Past medical history; Diagnoses } \\
\text { and comorbidities; Physical activity profile (5) }\end{array}$ \\
\hline Remote support & $\begin{array}{l}\text { Virtual and real-time monitoring: Telemetric } \\
\text { portable recording devices; Mobile apps (5) }\end{array}$ \\
\hline
\end{tabular}

only supervised exercises according to AACVPR considerations for resuming in-center Cardiac and Pulmonary Rehabilitation Program Services (10).

\section{Acknowledgments}

We feel obliged to acknowledge Nima Jalali for proofreading the final manuscript.

\section{Footnotes}

Authors' Contribution: R. N., M. G. N., and A. S. Kh. developed the original idea, S. N., M. H. P. Sh., and R. N. wrote the manuscript.

Conflict of Interests: There was no conflict of interests. Funding/Support: It was not declared by the authors.

\section{References}

1. Tan W, Aboulhosn J. The cardiovascular burden of coronavirus disease 2019 (COVID-19) with a focus on congenital heart disease. Int J Cardiol. 2020;309:70-7. doi:10.1016/j.ijcard.2020.03.063. [PubMed:32248966]. [PubMed Central: PMC7102656].

2. World Health Organization. Coronavirus disease (COVID-19) advice for the public. Geneva, Swizerland: World Health Organization; 2021, [cited 4th Jun 2020]. Available from: https://www.who.int/ emergencies/diseases/novel-coronavirus-2019/advice-for-public.

3. Lippi G, Henry BM, Sanchis-Gomar F. Physical inactivity and cardiovascular disease at the time of coronavirus disease 2019 (COVID-19). Eur J Prev Cardiol. 2020;27(9):906-8. doi: 10.1177/2047487320916823. [PubMed: 32270698]. [PubMed Central: PMC7717305].

4. Thompson W.ACSM's resource manual for Guidelines for exercise testing and prescription. Philadelphia, USA: Wolters Kluwer Health/Lippincott Williams \& Wilkins; 2009.

5. Thomas RJ, Beatty AL, Beckie TM, Brewer LC, Brown TM, Forman $\mathrm{DE}$, et al. Home-based cardiac rehabilitation: A scientific statement from the American Association of Cardiovascular and Pulmonary Rehabilitation, the American Heart Association, and the American College of Cardiology. J Am Coll Cardiol. 2019;74(1):133-53. doi: 10.1016/j.jacc.2019.03.008. [PubMed: 31097258]. [PubMed Central: PMC7341112].

6. Hwang R, Bruning J, Morris NR, Mandrusiak A, Russell T. Home-based telerehabilitation is not inferior to a centre-based program in patients with chronic heart failure: A randomised trial. J Physiother. 2017;63(2):101-7. doi: 10.1016/j.jphys.2017.02.017. [PubMed: 28336297].

7. Nourian R, Niyazi S, Nazarieh M, Sharafi SE, Pourgharib Shahi MH. IASEM-TUMS COVID-19 virtual pulmonary rehabilitation framework; exercise prescription for recovered COVID-19 patients. Asian J Sports Med. 2020;11(4). e107575. doi: 10.5812/asjsm.107575.

8. American Association of Cardiovascular \&amp; Pulmonary Rehabilitation. Guidelines for cardia rehabilitation and secondary prevention programs-(with web resource). Human Kinetics. 2013.

9. Williams MA, Balady GJ, Carlson JJ, Comoss P, Humphrey R, Lounsbury PF, et al. Guidelines for cardiac rehabilitation and secondary prevention programs. USA: Champaign Human Kinetics. 2004.

10. The American Association of Cardiovascular and Pulmonary Rehabilitation. Considerations for resuming in-center cardiac and pulmonary rehabilitation program services. Illinois, USA: AACVPR; 2020, [cited 16th Aug 2020]. Available from: https://www.corhealthontario.ca/AACVPR-Considerations-forResuming-In-Center-CR-PR-Program-Services_FINAL.pdf. 